\title{
Nonspecific Binding Domains in Lipid Membranes Induced by Phospholipase $\mathbf{A}_{2}$
}

\author{
Chia Yee Hong, Chung-Ta Han, and Ling Chao* \\ Department of Chemical Engineering, National Taiwan University, Taipei, Taiwan \\ Email: lingchao@ntu.edu.tw \\ Supplementary Information
}

Lipid membrane diffusion coefficients obtained by fluorescence recovery after photobleaching (FRAP). We used fluorescence recovery after photobleaching (FRAP) technique to examine the lipid membrane mobility. The FRAP technique uses laser to bleach fluorescent lipid molecules in a small region of the sample and the diffusion coefficients can be obtained by analyzing the fluorescence recovery with time in this small region. ${ }^{1-3}$ The recovery time evolution images of the photobleached spot were recorded by image processing program HCImage (Hamamatsu, Japan). The intensity recovery with time inside the region of interest was then processed and fitted by MATLAB (Mathworks Natick, MA) in order to obtain the diffusion coefficient of the SLB. The fitting algorithm we used for the two dimensional lateral diffusion coefficient was mainly based on the one developed by Axelrod et al. ${ }^{1}$ and the details are in the following paragraphs.

The measured fluorescence intensity was normalized by the following equation to subtract the fluorescence background and to make it fit to the modeling equation more easily.

$$
f_{e}(t)=\frac{F_{e}(t)-F_{e}(0)}{F_{e, \infty}(t)-F_{e}(0)}
$$

where $F_{e}(t)$ is the average intensity inside the region of interest which would recover with time, $F_{e}(0)$ is the average intensity inside the region of interest right after the lipid bilayers photobleached by laser and $\mathrm{F}_{\mathrm{e}, \infty}(\mathrm{t})$ is the average intensity inside the region of interest which assume to reach after infinite time. Considering the effect of photobleaching of fluorephores and fluctuation of intensity inside the whole observing sight, we took $\mathrm{F}_{\mathrm{e}, \infty}(\mathrm{t})$ as a function of time in order to eliminate the effect. The variation of $\mathrm{F}_{\mathrm{e}}$ (0) was found to be negligible comparing to $\mathrm{F}_{\mathrm{e}, \infty}(\mathrm{t})$, so we assume the value to be constant here. The calculated recovery percentages $\mathrm{f}_{\mathrm{e}}(\mathrm{t})$ would increase from zero to a maximum value, which would depend on the mobile fraction of the lipid bilayers. 
The normalized measured fluorescence intensity can be fitted to the theoretical normalized intensity derived from the transport model to obtain the diffusion coefficient parameter. For a bleached spot with its initial profile described by a Gaussian profile, the theoretical intensity with time, $F_{K}(t)$, can be obtained by solving mass transport equation.

$$
F_{K}(t)=\frac{q P_{0} C_{0}}{A} \sum_{n=0}^{\infty} \frac{(-K)^{n}}{n !\left[1+n\left(1+\frac{2 t}{\tau_{D}}\right)\right]}
$$

where $\mathrm{q}$ is the fluorophore quantum efficiency, $\mathrm{A}$ is the beam attenuation factor, $\mathrm{P}_{0}$ is the total laser power, $\mathrm{K}$ is bleaching parameter, $\mathrm{C}_{0}$ is the uniform fluorophore concentration before bleaching, and $\tau_{\mathrm{D}}$ is the characteristic time of diffusion. The theoretical normalized intensity can be expressed as:

$$
f_{k}(t)=\frac{\mathrm{F}_{\mathrm{K}}(\mathrm{t})-\mathrm{F}_{\mathrm{K}}(0)}{\mathrm{F}_{\mathrm{K}, \infty}(\mathrm{t})-\mathrm{F}_{\mathrm{K}}(0)}=f_{m} \frac{\sum_{n=0}^{\infty} \frac{(-K)^{n}}{n !\left[1+n\left(1+\frac{2 t}{\tau_{D}}\right)\right]}-\frac{1-e^{-K}}{K}}{1-\frac{1-e^{-K}}{K}}
$$

Note that the bleaching parameter, $\mathrm{K}$, can be obtained by fitting the initial bleaching profile to a Gaussian profile. $f_{m}$ is the mobile fraction. When the entire membrane is fluid and mobile, $f_{m}$ is set as one. When there exists immobile region, $\mathrm{f}_{\mathrm{m}}$ is set as a fitted parameter.

$$
\mathrm{C}(\mathrm{r}, \mathrm{t}=0)=C_{0} \exp \left[-K \exp \left(-2 r^{2} / w^{2}\right)\right]
$$

where $\mathrm{C}(\mathrm{r}, \mathrm{t}=0)$ is the fluorophore concentration profile at position $\mathrm{r}$ and time $\mathrm{t}=0, \mathrm{C}_{0}$ is the uniform fluorophore concentration before bleaching. Bleaching parameter, $\mathrm{K}$, and half-width, w, are the fitted parameters to be obtained.

By fitting the experimentally measured $f_{e}(t)$ to the theoretically obtained $f_{k}(t)$, we can obtain the fitted parameter $\tau_{\mathrm{D}}$ and the diffusion coefficient $\mathrm{D}$ can be calculated from the following equation: $\mathrm{D}=\frac{w^{2}}{4 \tau_{D}}$ 
(a) $100 / 0 \mathrm{DOPC} / \mathrm{DPPC}$

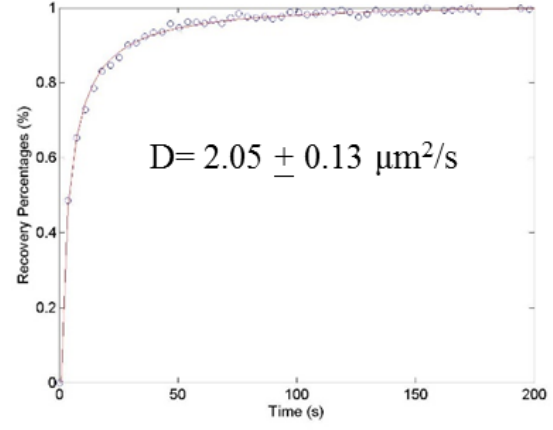

(d) $25 / 75 \mathrm{DOPC} / \mathrm{DPPC}$

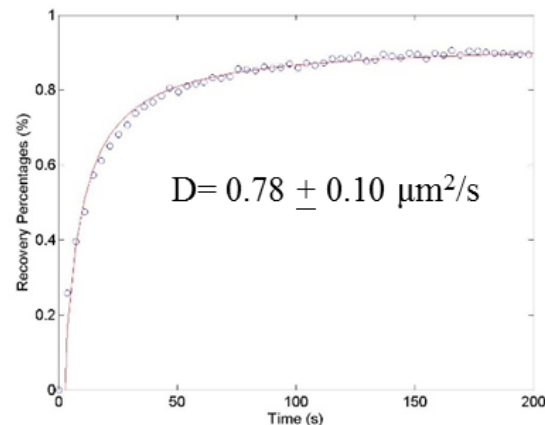

(c) 50/50 DOPC/DPPC
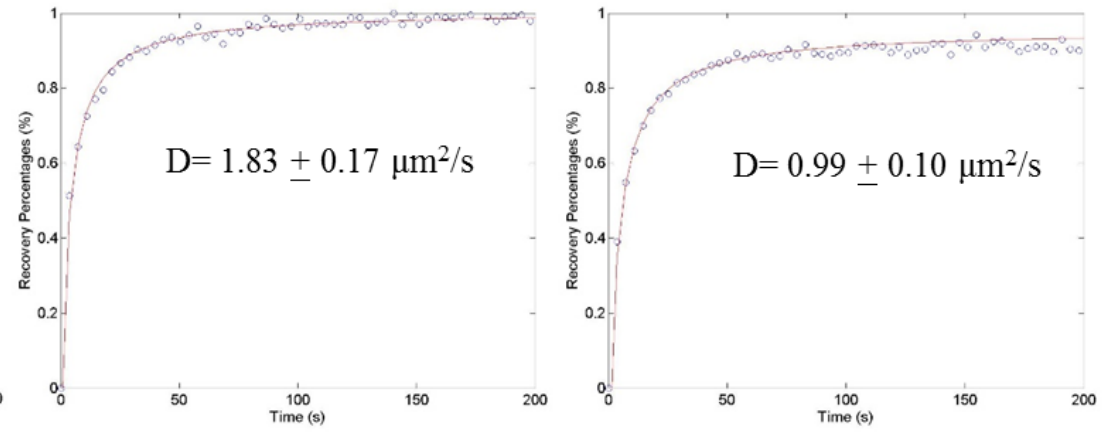

(e) $0 / 100$ DOPC/DPPC

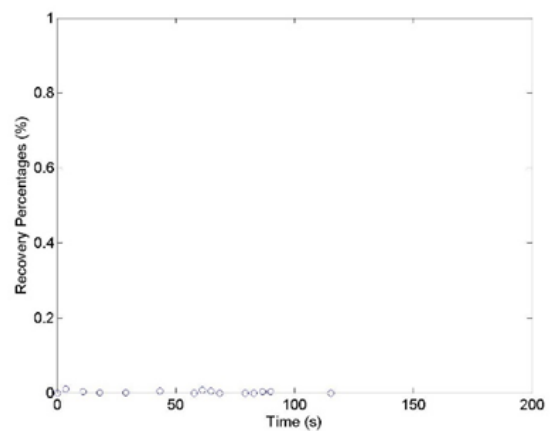

Figure S1. The FRAP data obtained at $25^{\circ} \mathrm{C}$. Red line: fitted curve used to obtain lipid membrane diffusion coefficient (D). The 0/100 DOPC/DPPC membrane almost had no recovery in the interested time duration and therefore the diffusion coefficient is not obtained here.

(a) $100 / 0$ DOPC/DPPC

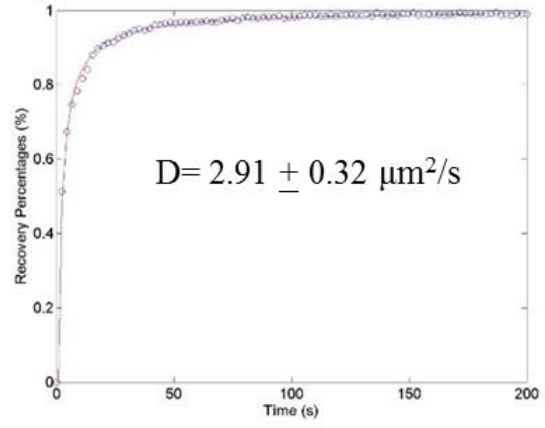

(b) $75 / 25$ DOPC/DPPC

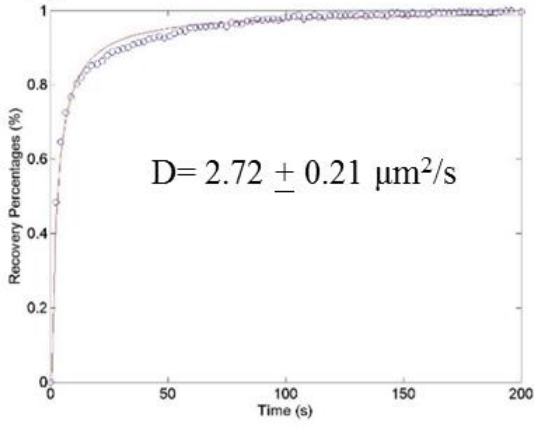

(c) $50 / 50 \mathrm{DOPC} / \mathrm{DPPC}$

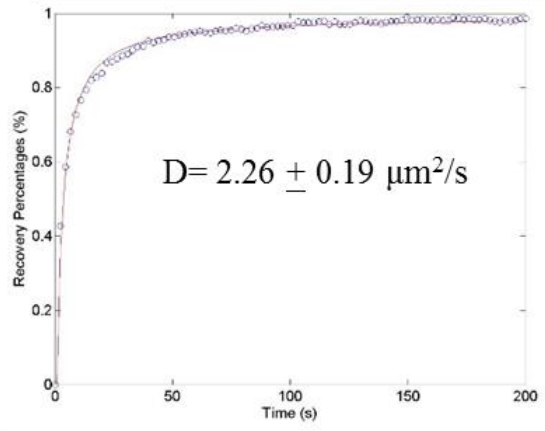

(d) $25 / 75$ DOPC/DPPC

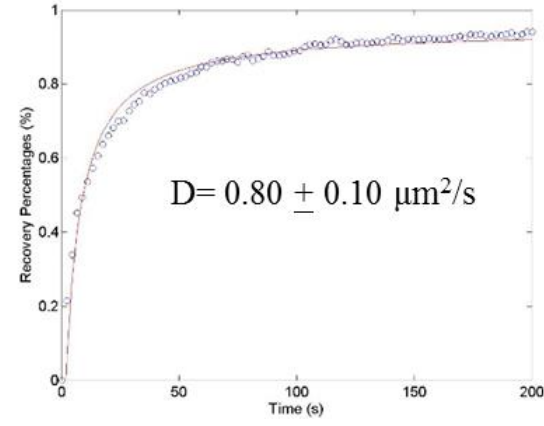

(e) $0 / 100 \mathrm{DOPC} / \mathrm{DPPC}$

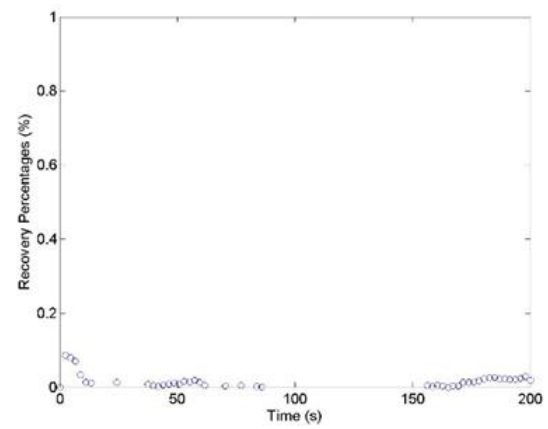


Figure S2. The FRAP data obtained at $37{ }^{\circ} \mathrm{C}$. Red line: fitted curve used to obtain lipid membrane diffusion coefficient (D). The 0/100 DOPC/DPPC membrane almost had no recovery in the interested time duration and therefore the diffusion coefficient is not obtained here.

\section{Morphology evolution induced by PLA2 at $37{ }^{\circ} \mathrm{C}$}

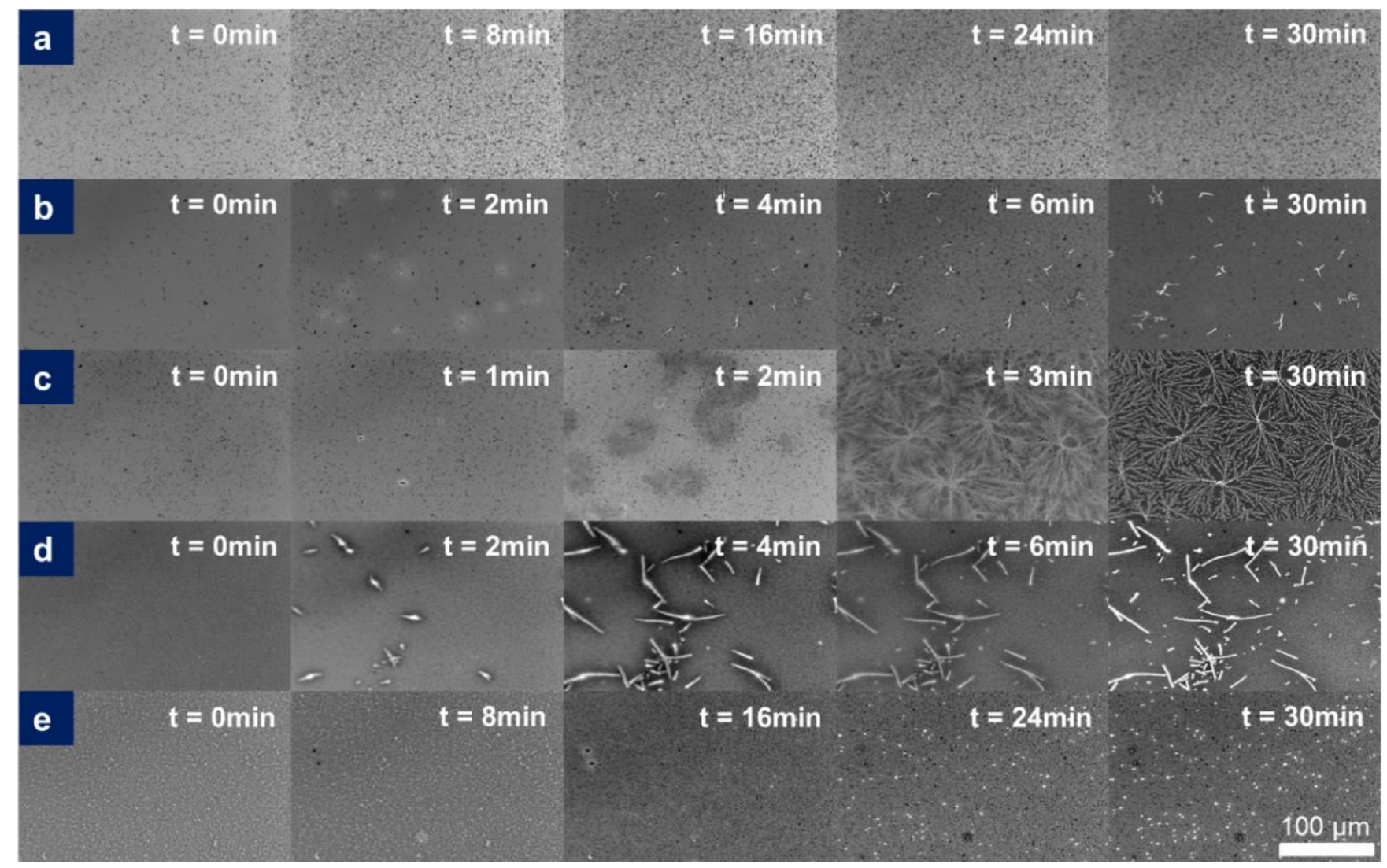

Figure S3. Morphology evolution images of SLBs with different molar ratios of DOPC/DPPC after addition of $\mathrm{PLA}_{2}$ at $0.009 \mathrm{unit} / \mu \mathrm{L}$ at $37^{\circ} \mathrm{C}$. Texas Red DHPE $(0.5 \mathrm{~mol} \%)$ was incorporated into SLBs to reveal the morphology change. (a) 100/0 DOPC/DPPC. (b) 75/25 DOPC/DPPC, (c) 50/50 DOPC/DPPC, (d) 25/75 DOPC/DPPC, and (e) 0/100 DOPC/DPPC.

\section{PLA2-induced domains as binding templates for PLA2 revealed using fluorescently labeled PLA2.}

To determine the location of $\mathrm{PLA}_{2}$ during the phase change and to eliminate the possibility that the new domains were formed by the hydrolysis of Texas Red DHPE, we performed experiments using labeled $\mathrm{PLA}_{2}$ (Alexa Fluor 488) and SLBs with no labeled lipids. Figure S4 shows the fluorescence images of nonlabeled SLBs with different molar ratios of DOPC/DPPC after the addition of Alexa Fluor 488-labeled 
$\mathrm{PLA}_{2}$ for $30 \mathrm{~min}$ before any image processing. The processed images to eliminate the uneven illuminated background are shown in Figure 2 in the main text.

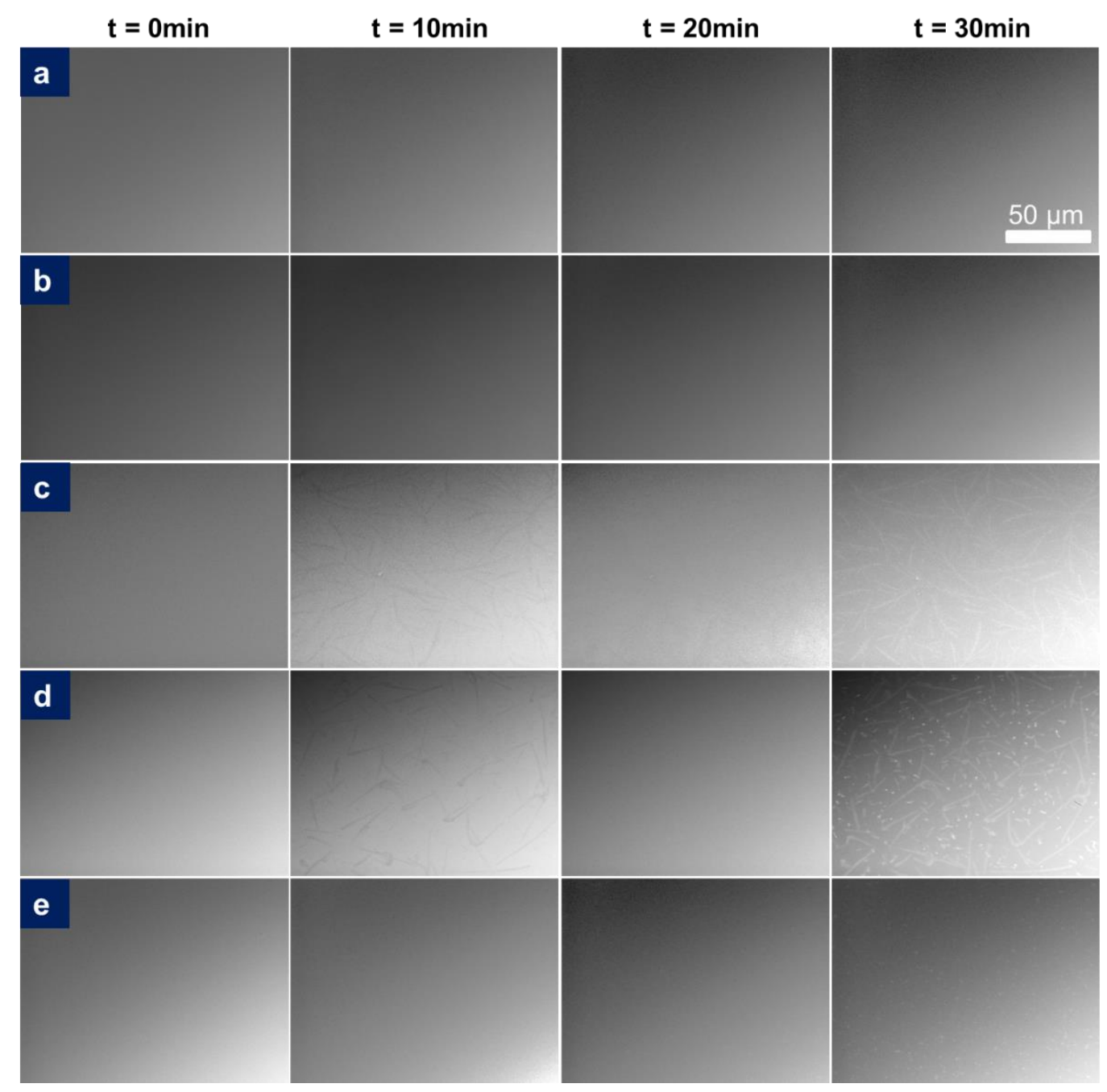

Figure S4. The unprocessed fluorescence images of nonlabeled SLBs with different molar ratios of DOPC/DPPC after the addition of Alexa Fluor 488-labeled PLA 2 for 30 min (approximately 0.009 unit/ $\mu \mathrm{L}$ ) at room temperature. (a) 100/0 DOPC/DPPC. (b) 75/25 DOPC/DPPC, (c) 50/50 DOPC/DPPC, (d) 25/75 DOPC/DPPC, and (e) 0/100 DOPC/DPPC.

Fluorescence images of the experiment with labeled PLA2 and the membrane labeled with Texas Red DHPE. Figure S5 shows fluorescence images taken after 30 min incubation of Alexa-488 labeled $\mathrm{PLA}_{2}$ and the membrane labeled with Texas Red DHPE. Texas Red DHPE may compete with PLA for $^{2}$ binding to the template and therefore influence the binding of PLA 2 to the template; consequently, we demonstrated the results with labeled $\mathrm{PLA}_{2}$ and nonlabeled membranes in the main text (Figure 2). 
However, the images show that the domains revealed using labeled $\mathrm{PLA}_{2}$ were at the same location as those revealed using Texas Red DHPE.

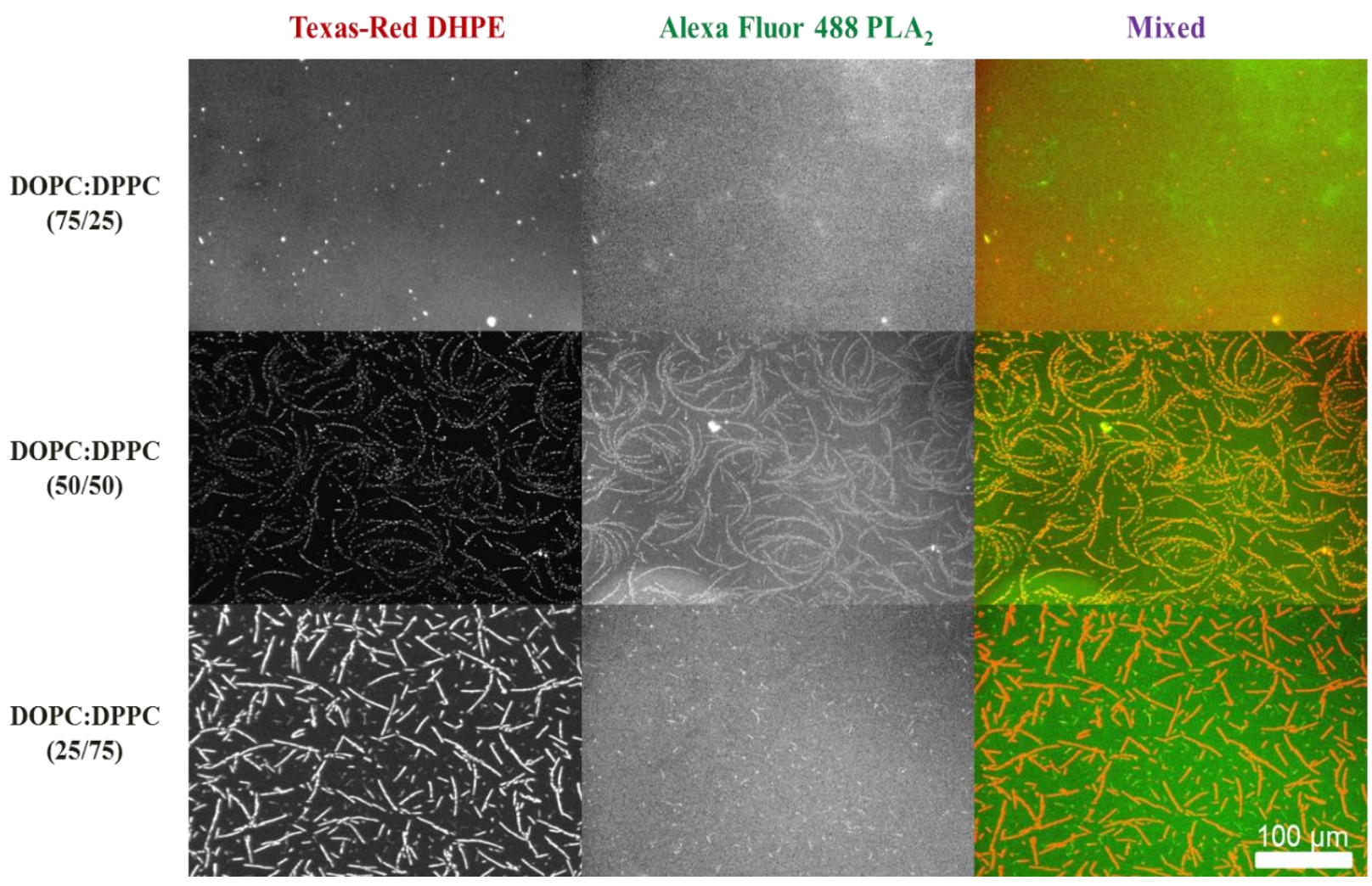

Figure. S5. Fluorescence images of DOPC/DPPC Texas-Red DHPE phospholipid bilayers in different molar ratio hydrolyzed by labeled PLA 2 (Alexa Fluor 488) one day later. The red color is from Texas-Red DHPE, the green color is from Alexa Fluor 488. All experiments were treated with 0.009 unit/ $\mu \mathrm{L}$ of PLA 2 at room temperature.

Similar domains can be observed after the membrane (50/50 DOPC/DPPC) is treated with 0.004 unit/ml porcine pancreatic PLA2.

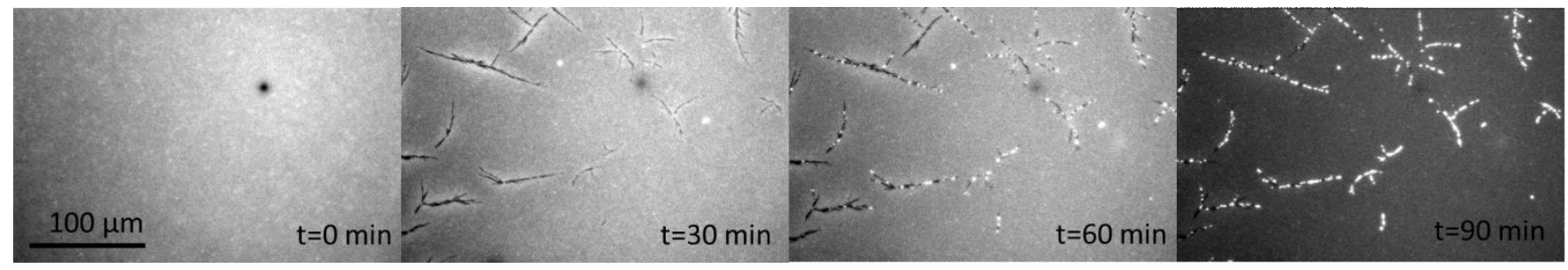


Figure S6. The membrane morphology of 50/50 DOPC/DPPC after treated with 0.004 unit/ $\mu \mathrm{L}$ porcine pancreatic $\mathrm{PLA}_{2}$ at room temperature (around $\left.25^{\circ} \mathrm{C}\right)$. Texas Red DHPE $(0.5 \mathrm{~mol} \%)$ was incorporated into SLBs to reveal the morphology change.

PLA2 binding to the domains can be washed off. Figure S7(a) shows the fluorescence images of labeled PLA2 (Alexa Fluor 488) after the 50/50 DOPC/DPPC lipid membrane was treated with 0.009 unit/ $\mu \mathrm{L}$ of labeled $\mathrm{PLA}_{2}$ at room temperature for 1 hour. Figure S7(b) shows the image taken right after the sample was extensively washed with a $\mathrm{Ca}^{2+}$-HEPES buffer $(10 \mathrm{mM}$ HEPES, $123 \mathrm{mM} \mathrm{NaCl}$, and $2.5 \mathrm{mM}$ $\left.\mathrm{CaCl}_{2}, \mathrm{pH}=7.4\right)$. $\mathrm{PLA}_{2}$ can be washed off from the domains while some of PLA 2 was still left on the rest of the lipid membrane. Since our AFM study in the main text shows that the 3-D structured domains still remained after the extensive wash, the labeled PLA 2 image result here shows that PLA 2 probably does not compose the 3-D structured domains.

(a)

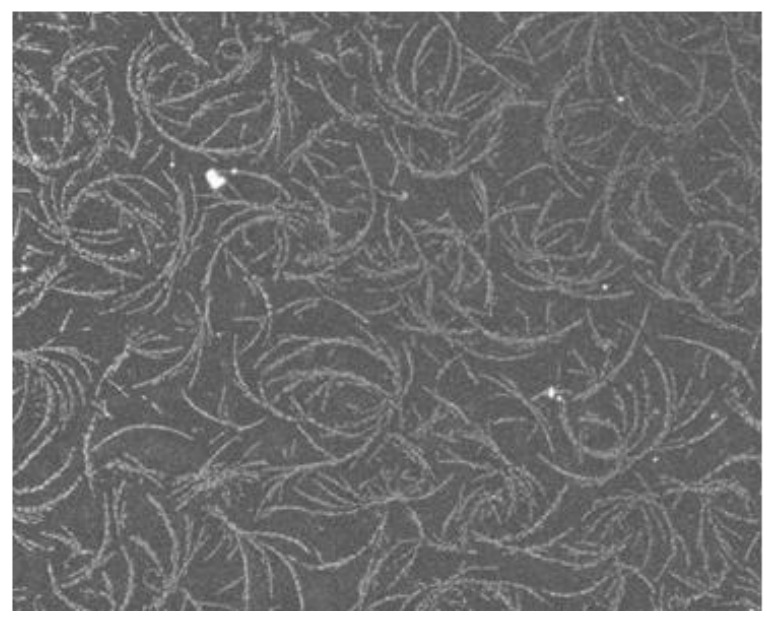

(b)

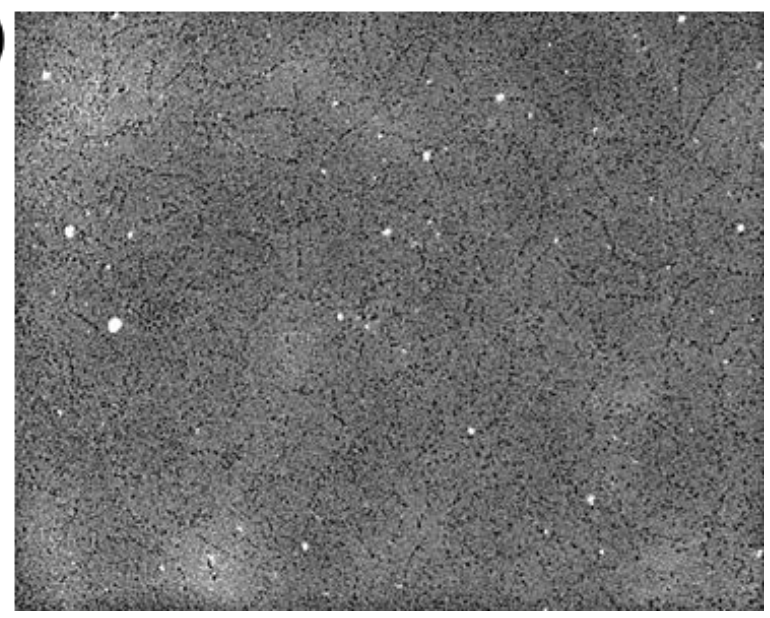

Figure S7. Fluorescence images of labeled PLA 2 (Alexa Fluor 488) in 50/50 DOPC/DPPC lipid membranes.(a) After the membrane was treated with 0.009 unit/ $\mu \mathrm{L}$ of labeled $\mathrm{PLA}_{2}$ at room temperature for 1 hour; (b) after the membrane in (a) was extensively washed with a $\mathrm{Ca}^{2+}$-HEPES buffer (10 mM HEPES, $123 \mathrm{mM} \mathrm{NaCl}$, and $\left.2.5 \mathrm{mM} \mathrm{CaCl}_{2}, \mathrm{pH}=7.4\right)$.

Examining whether calcium ion is required for the 3-D layer structure domain to be maintained. We extensively washed the sample with EDTA solution (10 mM HEPES, $123 \mathrm{mM} \mathrm{NaCl}$, and $1 \mathrm{mM}$ EDTA, $\mathrm{pH}=7.4)$ after PLA2 were incubated with the membrane (50/50 DOPC/DPPC) for $30 \mathrm{~min}$. The domains still remained on the support after the EDTA treatment, suggesting that the domains do not 
require $\mathrm{Ca}^{2+}$ to be maintained and the alignment of the products in the domain may already provide enough energy reward for the domain to be thermodynamically stable without the existence of $\mathrm{Ca}^{2+}$.
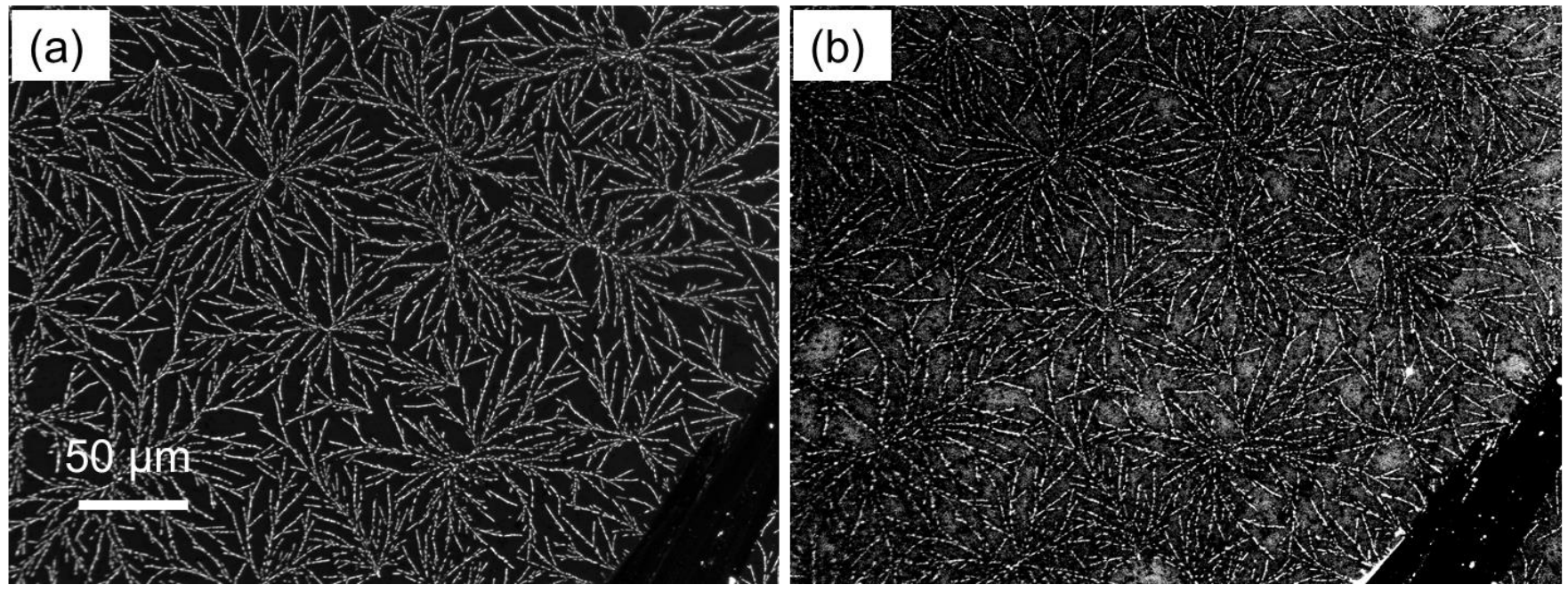

Figure S8. (a) The membrane morphology of 50/50 DOPC/DPPC after treated with 0.009 unit/ $\mu \mathrm{L}_{\mathrm{PLA}}$ at room temperature (around $25^{\circ} \mathrm{C}$ ) for $30 \mathrm{~min}$. Texas Red DHPE $(0.5 \mathrm{~mol} \%)$ was incorporated into SLBs to reveal the morphology change. (b) The membrane morphology after the sample was extensively washed with $10 \mathrm{mM}$ HEPES, $123 \mathrm{mM} \mathrm{NaCl}$, and $1 \mathrm{mM}$ EDTA, $\mathrm{pH}=7.4$. The scratch on the bottom right is to show the background fluorescence intensity.

\section{Reference}

1. Axelrod, D.; Koppel, D. E.; Schlessinger, J.; Elson, E.; Webb, W. W., Mobility measurement by analysis of fluorescence photobleaching recovery kinetics. Biophysical Journal 1976, 16, (9), 1055-1069.

2. Ratto, T. V.; Longo, M. L., Obstructed Diffusion in Phase-Separated Supported Lipid Bilayers: A Combined Atomic Force Microscopy and Fluorescence Recovery after Photobleaching Approach. Biophysical Journal 2002, 83, (6), 3380-3392.

3. Kang, M.; Day, C. A.; Drake, K.; Kenworthy, A. K.; DiBenedetto, E., A generalization of theory for twodimensional fluorescence recovery after photobleaching applicable to confocal laser scanning microscopes. Biophysical Journal 2009, 97, (5), 1501-1511. 